\title{
Comparative Assessment of LPV-based Predictive Control Strategies for a Pasteurization Plant
}

\author{
Fatemeh Karimi Pour \\ Institut de Robòtica i Informàtica \\ Industrial (CSIC-UPC), \\ Barcelona, Spain \\ Email: fkarimi@iri.upc.edu
}

\author{
Vicenç Puig \\ Institut de Robòtica i Informàtica \\ Industrial (CSIC-UPC), \\ Barcelona, Spain \\ Email: vpuig@iri.upc.edu
}

\author{
Carlos Ocampo-Martinez \\ Senior Member, IEEE \\ Institut de Robòtica i Informàtica \\ Industrial (CSIC-UPC), \\ Barcelona, Spain \\ Email: cocampo@iri.upc.edu
}

\begin{abstract}
This paper presents a comparative study of three different approaches to design Model Predictive Control (MPC) strategies for a pasteurization plant using Linear Parameter Varying (LPV) models. The first two methods consider the LPV model in the design of the MPC controller in two different manners. The last approach uses a Robust MPC controller for taking parameter variations of the LPV model into account. It is assumed that the disturbances are unknown but bounded and the zonotopic set representation is used for modeling the uncertainty. In addition, a comprehensive comparison of the closed-loop performance accounting the proposed approaches is carried out through a high-fidelity simulator of a utility-scale pasteurization plant.
\end{abstract}

\section{INTRODUCTION}

In modern times of industrialization, investigation about complex dynamical systems is one of the important issues in the industrial framework. Controlling these complex systems is one of the most important problems in control engineering, but also one of the most challenging. One of the main, successful and widespread advanced control methodologies in industrial processes is Model Predictive Control (MPC) [1]. The reason for its success is its flexibility, and its ability to take constraints on the states and/or input directly into account [2]. Basically, MPC uses a model of the system to predict its future behavior in order to compute appropriate input signals to control the system states/outputs. To do so, an input sequence is obtained by optimizing a finite-horizon cost function at each time instant, based on an explicit model of the process and state measurements/estimations. Only the first element of the optimal sequence is applied to the plant and the procedure is repeated at the next time instant with new measurements while the input sequence is updated [3]. This approach is called receding horizon control.

MPC based on linear models is typically used in process control where the on-line optimization problem can be formulated as a convex optimization problem by either linear programming or quadratic programming. This assumption is suitably considered for typical processes. However, most of the real systems show nonlinear behaviours that can be approximated by polytopic linear uncertain models. In order to reduce the conservativeness, the idea of controlling nonlinear systems considering linear parameter varying (LPV) models has been widely investigated in the literature [4], [5]. LPV models are a class of linear models whose state-space matrices depend on a set of time-varying parameters, which take their values into pre-specified sets [6]. The advantage of this class of models is that it embeds the system non-linearities into the varying parameters, which make the non-linear system become a linear-like one with varying parameters [7]. In quasi-LPV models, the varying parameters can be functions of states, inputs or outputs, to make a further distinction with respect to pure LPV models, where the varying parameters only depend on exogenous signals. In this way, LPV models allow applying powerful linear design tools to complex nonlinear models. The predictive control of LPV models can be addressed considering the parameter variation as uncertainty and using the concept of ellipsoidal invariant sets [8]. However, this approach can lead to conservative results. To reduce the conservativeness, the class of linear parameter-dependent Lyapunov functions has been introduced [9]. Then, a linear scheduled controller is computed at every time instant. The control performance improvements are remarkable at the price of an increased number of linear matrix inequality (LMI) conditions. In addition, in [10] the special class of non-linear parameter-dependent Lyapunov functions is used and a non-linear scheduled control law is obtained which, leads to further improve the control performance.

A pasteurization system includes typical behaviors of many industrial processes, such as complex dynamical models with nonlinearities, which imply important challenges when a suitable controller should be designed [11]. Regarding its control, the regulation of both water and milk temperatures by using MPC is reported in [12], where transient behaviors have been suitably handled with respect to other control techniques such as cascade generic model control (GMC) strategy. A recent study by [11] considered different control topologies based on MPC that aim to the minimization of the energy consumption.

In this paper, three different strategies for designing MPC controllers using LPV models are proposed for a pasteurization plant. The first strategy is an MPC controller based on a pureLPV model. In the second strategy, an MPC controller based on a quasi-LPV model is proposed by considering that the scheduling variable depends on the input of the system. The last strategy uses a Robust MPC controller with bounded disturbances and considers parameters variation in the LPV model as uncertainty. Finally, a comprehensive comparison between the performance of the different approaches is carried out by using a high-fidelity simulator of a utility-scale pasteurization plant.

The remainder of this paper is organized as follows. 


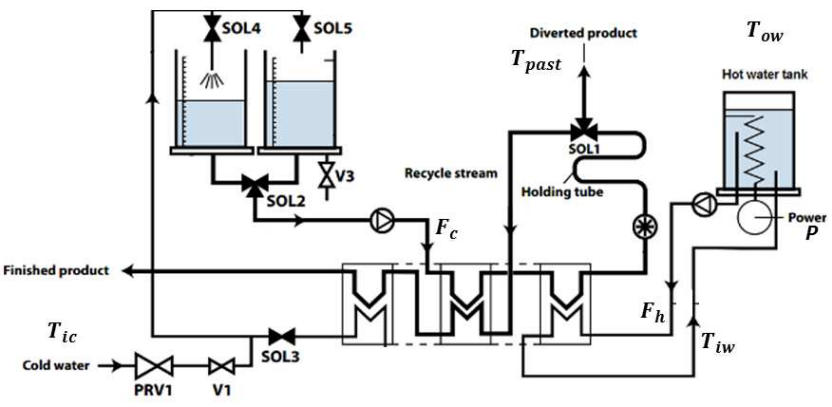

Fig. 1: Plant scheme.

Section II presents a pasteurization plant and describes the LPV model and the control objectives of the pasteurization plant. Section III describes the three MPC controller strategies. Section IV presents and compares the results obtained with the different strategies. Finally, in Section V, the conclusions of this work are drawn and some research lines for future work are proposed.

\section{PROBLEM STATEMENT}

\section{A. Pasteurization Model}

The considered pasteurization process is the small-scale plant PCT-23 MKII, manufactured by Armfield (UK) [13]. This laboratory plant is a small version $(1.2 \mathrm{~m}, 0.6 \mathrm{~m}, 0.6 \mathrm{~m})$ of an industrial pasteurization process. The High-Temperature Short-Time (HTST) approach is generally accepted as the industry standard for the pasteurization process [14]. In this process, the goal is to heat and preserve the product, which is typically a liquid, at a predetermined temperature for a minimum time. According to Fig. 1, the liquid is pumped at a predetermined flow rate from one of two storage tanks to the heat exchanger indirectly. The water heat is transferred to the product inside the first phase of the heat exchanger, which is called regenerator. The raw product is heated to an intermediate temperature by using missed energy from the pasteurized product. Then, in the second phase, this product is heated from that middle temperature to the full pasteurization temperature while using a hot-water flow $\left(F_{h}\right)$ coming from a closed circuit with a heater. The temperature at the output of the holding tube $\left(T_{\text {past }}\right)$ is used to monitor the product temperature after the pasteurization process. Finally, the product is cooled in the third phase of the heat exchanger, where the remaining heat is removed to the inlet product. This last phase does not add anything new to the model, then it was not considered in this paper.

The block diagram of the model used in this article has been established in [15] (see Fig. 2). For the modelling purpose, the whole pasteurization system can be divided into three subsystems that are a hot water tank, heat exchanger and holding tube. To model the whole pasteurization plant, models of these subsystems are obtained and, at the end, they are connected together. The mathematical models of the subsystems are obtained from the experimental data reported in [15]. Accordingly, identified models obtained as transfer functions are suitably stated by their equivalent controllable realizations in state space, with varying parameters according

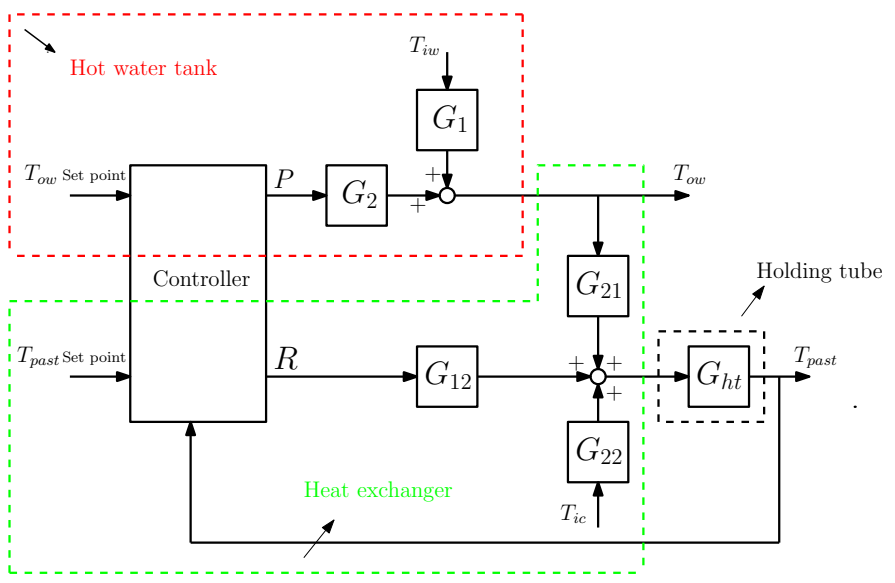

Fig. 2: Block diagram of the pasteurization plant

to the hot water flow, $F_{h}$, as exogenous input and the hot/cold water flow ratio, $R$, as the input of the system. Observing Fig. 2 , it can be seen the controlled inputs are the power of the electrical heater, $P$, and hot/cold flow ratio $\left(R=F_{h} / F_{c}\right)$. The input temperature of the water heater, $T_{i w}$, and temperature of cold water, $T_{i c}$ are measured non-controlled inputs (disturbances). The temperature of the hot water tank $T_{\text {ow }}$ and the pasteurization temperature of the process $T_{\text {past }}$ are the first and second outputs of this system, respectively.

Collecting all the information above, the following statespace LPV model can be written:

$$
\begin{aligned}
& \dot{x}=A\left(\theta_{t}\right) x_{t}+B\left(\theta_{t}\right) u_{t}, \\
& y=C\left(\theta_{t}\right) x_{t}+D\left(\theta_{t}\right) u_{t},
\end{aligned}
$$

where the continuous-time variable is denoted by $t \in \mathbb{R}_{\geq 0}$ and $x \in \mathbb{R}^{n_{x}}$ is the system state vector, $u \in \mathbb{R}^{n_{u}}$ is the vector of manipulated variables, $y \in \mathbb{R}^{n_{y}}$ is the vector of measured variables and the system matrices including the varying parameters in function of the scheduling variables $\left(F_{h}\right.$ and $R$ ) are

$$
\begin{aligned}
& A=\left[\begin{array}{cccccc}
\frac{-1}{\tau_{1}\left(F_{h}(t)\right)} & 0 & 0 & 0 & 0 & 0 \\
0 & \frac{-1}{\tau_{2}\left(F_{h}(t)\right)} & 0 & 0 & 0 & 0 \\
\frac{K_{21}(R(t))}{\tau_{21}(R,(t))} & \frac{K_{21}(R(t))}{\tau_{21}(R(t))} & \frac{-1}{\tau_{21}(R(t))} & 0 & 0 & 0 \\
0 & 0 & 0 & \frac{-1}{\tau_{12}(R(t))} & 0 & 0 \\
0 & 0 & 0 & 0 & \frac{-1}{\tau_{22}(R(t))} & 0 \\
0 & 0 & \frac{K_{h t}}{\tau_{h t}} & \frac{K_{h t}}{\tau_{h t}} & \frac{K_{h t}}{\tau_{h t}} & \frac{-1}{\tau_{h t}}
\end{array}\right] \\
& B=\left[\begin{array}{cc}
0 & 0 \\
\frac{K_{2}\left(F_{h}(t)\right)}{\tau_{2}\left(F_{h}(t)\right)} & 0 \\
0 & 0 \\
0 & \frac{K_{12}(R(t))}{\tau_{12}(R(t))} \\
0 & 0 \\
0 & 0
\end{array}\right] \quad E=\left[\begin{array}{cc}
\frac{K_{1}\left(F_{h}(t)\right)}{\tau_{1}\left(F_{h}(t)\right)} & 0 \\
0 & 0 \\
0 & 0 \\
0 & 0 \\
0 & \frac{K_{22}(R(t))}{\tau_{22}(R(t))} \\
0 & 0
\end{array}\right], \\
& C=\left[\begin{array}{llllll}
1 & 1 & 0 & 0 & 0 & 0 \\
0 & 0 & 0 & 0 & 0 & 1
\end{array}\right],
\end{aligned}
$$

where $K$ is the static gain and $\tau$ is the time constant of the transfer functions of the subsystems. The indexes of $K$ and $\tau$ are related to the transfer functions of the each subsystems from the whole pasteurization system (see Fig. 2). For control purposes, this model is discretized with sampling time $T_{s}=4 \mathrm{~s}$ 
and written as follows:

$$
\begin{aligned}
x_{k+1} & =A\left(\theta_{k}\right) x_{k}+B\left(\theta_{k}\right) u_{k}, \\
y_{k} & =C\left(\theta_{k}\right) x_{k}+D\left(\theta_{k}\right) u_{k},
\end{aligned}
$$

where the discrete-time is denoted by $k \in \mathbb{Z}_{>0}$. The system matrices $A\left(\theta_{k}\right) \in \mathbb{R}^{n_{x} \times n_{x}}, B\left(\theta_{k}\right) \in \mathbb{R}^{n_{x} \times n_{u}}, C\left(\theta_{k}\right) \in$ $\mathbb{R}^{n_{y} \times n_{x}}$ and $D\left(\theta_{k}\right) \in \mathbb{R}^{n_{y} \times n_{u}}$ are assumed to depend on the parameter vector $\theta_{k}:=\left[\theta_{1, k}, \theta_{2, k}, \ldots, \theta_{N, k}\right]^{T} \in \mathbb{R}^{N}$, which belongs to a convex polytope $\Theta$ defined by

$$
\Theta:=\left\{\theta_{k} \in \mathbb{R}^{N} \mid \sum_{j=1}^{N} \theta_{j, k}=1, \theta_{j, k} \geq 0\right\},
$$

where $N$ is the number of vertices of the polytope. Hence, as $\theta_{k}$ varies inside the convex polytope $\Theta$, the matrices of the system (2) vary inside a corresponding polytope $\Psi$, which is defined by the convex hull $(\mathrm{Co})$ of $N$ local matrix vertices $\left[A_{i}, B_{i}, C_{i}, D_{i}\right], i \in[1, . ., N]$, i.e.,

$$
\begin{gathered}
\Psi:=\operatorname{Co}\left\{\left[\begin{array}{llll}
A_{1} & B_{1} & C_{1} & D_{1}
\end{array}\right],\left[\begin{array}{llll}
A_{2} & B_{2} & C_{2} & D_{2}
\end{array}\right], \ldots,\right. \\
{\left[\begin{array}{llll}
A_{N} & B_{N} & C_{N} & D_{N}
\end{array}\right],}
\end{gathered}
$$

and the matrices of the system (2) can be rewritten as

$$
\begin{array}{ll}
A\left(\theta_{k}\right)=\sum_{j=1}^{N} \theta_{j, k} A_{j}, & B\left(\theta_{k}\right)=\sum_{j=1}^{N} \theta_{j, k} B_{j}, \\
C\left(\theta_{k}\right)=\sum_{j=1}^{N} \theta_{j, k} C_{j}, & D\left(\theta_{k}\right)=\sum_{j=1}^{N} \theta_{j, k} D_{j} .
\end{array}
$$

\section{B. Control objectives}

The most important objective of the pasteurization process controller is to control and maintain the temperature of the processed product. First, the raw product must be retained in the holding tube during the required pasteurization time, and second the pasteurization temperature must be reached and maintained as close as possible to a given value. In fact, the necessity of a significant control for the process arises from the saving in energy, product and time if an accurate tracking of that reference temperature is performed [15] while trying to minimize the electric heater power of the hot water tank.

The LPV model of the pasteurization plant (1) demonstrates the inherent nonlinearity and time-varying features of the system, also allows controller designers to use linear-like control theory for nonlinear system control. This paper studies the feasibility to design MPC controllers that use this LPV model. In the following section, three strategies to design the MPC controller for a pasteurization plant based on the LPV model are described.

\section{PROPOSED LPV-BASED MPC STRATEGIES}

The MPC controller uses a dynamic model of the process to predict the output trajectories and performs a constrained online optimization to determine the optimal future input sequence. In this section, three different MPC strategies based on the LPV model of the pasteurization plant with respect to the situation of scheduling parameter are proposed.

\section{A. MPC based on the pure-LPV model}

The LPV model can be usually described by a polytopic family of the linear models, whose parameters vary according to a time-varying parameter vector, which is restricted to lie into a unit simplex. The time-varying parameters are scheduled online using the scheduling variable and then providing realtime information on the variations of the plant dynamics. In order to formalize the statement, the assumptions below are taken into account.

Assumption 1: The varying parameters of the system only depend on exogenous signal.

Assumption 2: The sequence of scheduling variables is available at every time instant $k$.

By considering Assumptions 1 and 2, the LPV model (2) can be represented by the following linear time-varying system:

$$
\begin{aligned}
x_{k+1} & =A_{k} x_{k}+B_{k} u_{k}, \\
y_{k} & =C_{k} x_{k}+D_{k} u_{k},
\end{aligned}
$$

where $A_{k}, B_{k}, C_{k}$ and $D_{k}$ belong to a convex polytope $\Psi$ defined in (4) while each model corresponds to a different operating point of the system at each time instant $k$. By assuming that both the time-varying parameter $\theta_{k}$ and the state $x_{k}$ are available, the following optimization problem is solved:

$$
\min _{\tilde{u}_{k}} J_{k}=\sum_{i=0}^{N_{p}-1}\left\|x_{i \mid k}\right\|_{w_{1}}^{P}+\left\|u_{i \mid k}\right\|_{w_{2}}^{P}
$$

subject to

$$
\begin{aligned}
& x_{i+1 \mid k}=A_{k} x_{i \mid k}+B_{k} u_{i \mid k}, \quad \forall i \in\left\{0, \ldots, N_{p}-1\right\} \\
& u_{i \mid k} \in \mathbb{U}, \quad \forall i \in\left\{0, \ldots, N_{p}-1\right\}, \\
& x_{i \mid k} \in \mathbb{X}, \quad \forall i \in\left\{1, \ldots, N_{p}\right\}, \\
& x_{N_{p} \mid k} \in \mathbb{X}_{f}, \\
& x_{0 \mid k}=x_{k} .
\end{aligned}
$$

where $w_{1} \in \mathbb{R}^{n_{x} \times n_{x}}$ and $w_{2} \in \mathbb{R}^{n_{u} \times n_{u}}$ are positive definite weighting matrices that establish the trade-off between state and the control input effort, respectively. The superindex $P$ denotes the norm used. $\tilde{u}_{k}=\left[u_{0 \mid k}, u_{1 \mid k+1}, \ldots, u_{N_{p}-1 \mid k}\right]$ is the optimization vector at time $k$ and $x_{i \mid k}$ is the predicted state at time $i$, with $i=0, \ldots, N_{p}$, obtained by starting from the state $x_{0 \mid k}=x_{k}$ and applying to system (6) the input sequence, $\tilde{u}_{k} \cdot \mathbb{X}$ and $\mathbb{U}$ define the set of acceptable states and inputs. Therefore, the optimization problem (7) is convex since the cost function is quadratic and the constraints are linear. Thus, it can be solved with efficient quadratic programming (QP) tools.

\section{B. MPC based on the quasi-LPV model}

As highlighted in the previous subsection, in an LPV model parameters vary according to some scheduling function and variable (external to the loop) inside a pre-specified set. However, in a quasiLPV model, the varying parameters can be functions of internal variables (i.e., the states, inputs or outputs). In other words, the model parameters are exactly known at the current time, but their future evolutions are uncertain and contained into the prescribed bounded sets.

In the case of the pasteurization plant, by considering that the scheduling parameter $\theta$ depends on the controlled inputs, the model (2) becomes a quasi-LPV model and the optimization problem (7) becomes a non-linear one. Here, taking into account that the dynamics of the pasteurization plant reach very fast the steady state temperature, 
Assumption 3 will be considered since the prediction horizon $N_{p}$ used is not that long.

Assumption 3: It can be assumed that the value of LPV parameters at the current time step is equal to the next step ahead and equal to the value estimated at the beginning of the MPC prediction horizon. Thus, $\theta_{0 \mid k} \simeq \theta_{1 \mid k} \simeq \theta_{2 \mid k} \simeq \ldots \simeq \theta_{N_{p}-1 \mid k}$ can be considered along $N_{p}$.

Consequently, the LPV model of the pasteurization is just updated from one MPC iteration to the next. But, a linear-time invariant (LTI) model is used along $N_{p}$ into the MPC controller. Thus, taking into account the model (2) and Assumption 3, the MPC optimization problem (7) can be rewritten as follows:

$$
\min _{\widetilde{u}_{k}} J_{k}=\sum_{i=0}^{N_{p}-1}\left\|x_{i \mid k}\right\|_{w_{1}}^{P}+\left\|u_{i \mid k}\right\|_{w_{2}}^{P},
$$

subject to

$$
\begin{aligned}
& x_{i+1 \mid k}=A\left(\theta_{i \mid k}\right) x_{i \mid k}+B\left(\theta_{i \mid k}\right) u_{i \mid k}, \quad \forall i \in\left\{0, \ldots, N_{p}-1\right\} \\
& u_{i \mid k} \in \mathbb{U}, \quad \forall i \in\left\{0, \ldots, N_{p}-1\right\}, \\
& x_{i \mid k} \in \mathbb{X}, \quad \forall i \in\left\{1, \ldots, N_{p}\right\}, \\
& x_{N_{p} \mid k} \in \mathbb{X}_{f}, \\
& x_{0 \mid k}=x_{k} \\
& \theta_{i \mid k}=\theta_{i \mid 0}, \quad \forall i \in\left\{0, \ldots, N_{p}-1\right\} .
\end{aligned}
$$

\section{RMPC based on LPV model with bounded disturbances}

The underlying idea of the last strategy is to employ a Robust MPC (RMPC) with bounded disturbance instead of using the standard MPC controller. In this case, the local matrix vertices $\left[A_{i}, B_{i}\right]_{i \in[1, . ., N]}$ in (5) are used instead and considered as a bounded disturbance. Thus, model (2) by including uncertainties can be reformulated as follows

$$
x_{k+1}=\left(A_{0} \pm \Delta A\right) x_{k}+\left(B_{0} \pm \Delta B\right) u_{k},
$$

where $A_{0}$ and $B_{0}$ are the nominal state-space of the system and $\Delta A$ and $\Delta B$ represent the uncertainty that is considering the set that bounds $A_{\theta_{k}}$ and $B_{\theta_{k}}$ in (5). If the uncertainties are located in the parameters, a vector of uncertain time-varying parameters $\theta_{k}$ of dimension $p$ with their values bounded by a compact set $\theta \in \Theta$ of a box type, i.e. $\Theta=\left\{\theta \in \mathbb{R}^{p} \mid \underline{\theta} \leq \theta \leq \bar{\theta}\right\}$, is introduced while vectors $\underline{\theta}$ and $\bar{\theta}$ determine the minimum and maximum possible uncertain parameters, respectively.

Modelling uncertainty is represented by bounding model parameters in intervals. The uncertain parameters are considered time varying. This type of model is known as an interval model. The model (9) can be transformed into

$$
x_{k+1}=A_{0} x_{k}+B_{0} u_{k}+d_{k},
$$

where $d_{k} \in \Delta A x_{k}+\Delta B u_{k}$ is the bounded uncertainty of the system (9). The computation of the bounds of $d_{k}$ is done based on the setmembership approach proposed by [16] and based on both zonotopes and the Kühn method [17]. Then, the model (9) corresponds to an LTI model with uncertainty. Therefore, by using a min-max robust MPC based on the LTI model can be applied. A min-max MPC approach seeks the optimal control strategy considering the worstcase realization of the disturbance. Thus, the RMPC controller can be designed as

$$
J_{k}=\min _{\tilde{u}_{k}} \max _{d_{k}} \sum_{i=0}^{N_{p}-1}\left\|x_{i \mid k}\right\|_{w_{1}}^{P}+\left\|u_{i \mid k}\right\|_{w_{2}}^{P}
$$

subject to

$$
\begin{aligned}
& x_{i+1 \mid k}=A x_{i \mid k}+B u_{i \mid k}+d_{i} \quad \forall i \in\left\{0, \ldots, N_{p}-1\right\} \\
& d_{i} \in \Delta A x_{i \mid k}+\Delta B u_{i \mid k}, \\
& u_{i \mid k} \in \mathbb{U}, \quad \forall i \in\left\{0, \ldots, N_{p}-1\right\} \\
& x_{i \mid k} \in \mathbb{X}, \quad \forall i \in\left\{1, \ldots, N_{p}\right\} \\
& x_{N_{p} \mid k} \in \mathbb{X} f \\
& x_{0 \mid k}=x_{k} .
\end{aligned}
$$

\section{Simulation RESUlts}

In this section, simulation results for the proposed strategies are presented and analysed in detail.

\section{A. Scenarios}

In order to test the behaviour of the proposed MPC strategies for the pasteurization plant, several simulations were carried out and the results obtained are presented in the following scenarios.

1) Scenario 1: pure-LPV model: As mentioned in Section III, when the parameter varying only depends on exogenous signals, the pasteurization plant can be represented by a pure-LPV model (see Section III-A). Therefore, for implementing the MPC based on the pure-LPV model, the scheduling variables should be related to exogenous signals as $F_{h}$. Due to the fact that the hot/cold water flow ratio, $R$ is an endogenous variable, by assuming that the flow $F_{c}$ is constant, the ratio $R$ depend also on $F_{h}$. In this scenario, the water heater input $T_{i w}$ was maintained constant at $\simeq 30{ }^{\circ} \mathrm{C}$ and the input temperature to the heat exchanger $T_{i c}$ (the raw temperature) will be maintained at $\simeq 20^{\circ} \mathrm{C}$. The control objective of the MPC controller for the pasteurizations plant is that the temperatures $T_{o w}$ and $T_{\text {past }}$ track their setpoint.

The MPC controller has been implemented and tested in simulation using a high-fidelity model for the pasteurization plant considering that the weighting matrices are $w_{1}=1, w_{2}=0.01$ while $N_{p}=5$. The state and input are constraints to be $0 \leq x_{i \mid k} \leq 100$ and $[0,0.2]^{\top} \leq u_{i \mid k} \leq[2000,3]^{\top}$.

In Fig. 3, the controlled input and the behavior of the controlled temperatures $T_{o w}$ and $T_{\text {past }}$ from the pasteurization plant under the MPC controller using a pure-LPV model is shown, while the controlled variables track the references. The response of pasteurization temperature $T_{\text {past }}$ is very quick, while the response of second output temperature $T_{o w}$ presents some overshoot.

2) Scenario 2: quasi-LPV model: In case that the varying parameters of the LPV model are expressed as a functions of an endogenous variable (in this case the input), it is not a pure-LPV model but a quasi-LPV one (see Section III-B). As already discussed, the process of pasteurization changes quite fast and the considered prediction $N_{p}$ is not that long. Consequently, Assumption 3 applies and the LPV model can be expressed as an LTI model in the MPC horizon. But from one iteration of the next, the model is adapted taking into account the change in the inputs that acts as scheduling variables for the varying parameters of the LPV model. The MPC controller was implemented with the same weighting matrix and prediction horizon considered in the first strategy.

Figure 4 presents the obtained simulation results of the MPC controller based on the quasi-LPV model of the pasteurization. As it can be seen in this figure, outputs of the system are tracking references while references are changed at time 200s. Nevertheless, there is some steady state error in the the response of the pasteurization temperature $T_{\text {past }}$. 

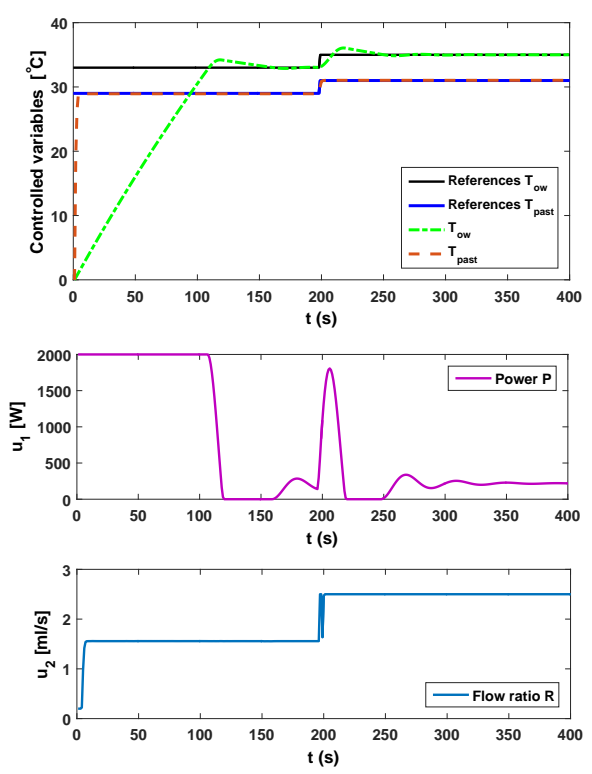

Fig. 3: Control of the output temperatures $\left(T_{o w}, T_{\text {past }}\right)$ and control inputs, MPC based on pure-LPV model of the pasteurization plant.

3) Scenario 3: uncertain LTI model: The last MPC controller is based on considering the varying parameters of the LPV model as uncertainties and then using robust MPC with bounded disturbances (see Section III-C). The disturbances represents the set of models corresponding to the different varying that are bounded using zonotopic sets. The robust MPC controller was implemented with the same weighting matrix and prediction horizon considered for the previous strategies. While, the bounded disturbance is estimated considering that nominal operating point is $F_{h} \in[155,694]$ for estimating disturbance bounds.

Figure 5 presents the bounds of first state considering model uncertainty and using using zonotopic sets. From Fig.6, it can be seen that the measured state (dotted black line) is inside the bounds obtained considering the model with uncertainty. Figure 7 shows the simulation results of robust MPC controller and the controlled inputs of system. It can be observed that the RMPC controller also achieves the set-points despite the uncertainty.

\section{B. Discussion and comparison}

Comparing the results of the different MPC strategies presented in Figs. 3, 4 and 7, it can be seen in that the objective of MPC is fulfilled since the controlled outputs follow the set-points. According to the Table II, the MPC controller based on the pure-LPV model has more knowledge and assumptions about the scheduling variables rather than others strategies. Therefore, it can be have better results regarding some criteria in theoretical analysis (see Table I). Table II shows the comparison of several control criteria considering the different strategies of applying an MPC based on LPV models to the pasteurization system.

\section{CONCLUSION}

In this paper, three strategies to design MPC controllers for a pasteurization plant based on a LPV model has been presented. Taking into account the information regarding the knowledge of the scheduling variables of the varying parameters, the first and second strategies of MPC controller based on pure-LPV and quasi-LPV
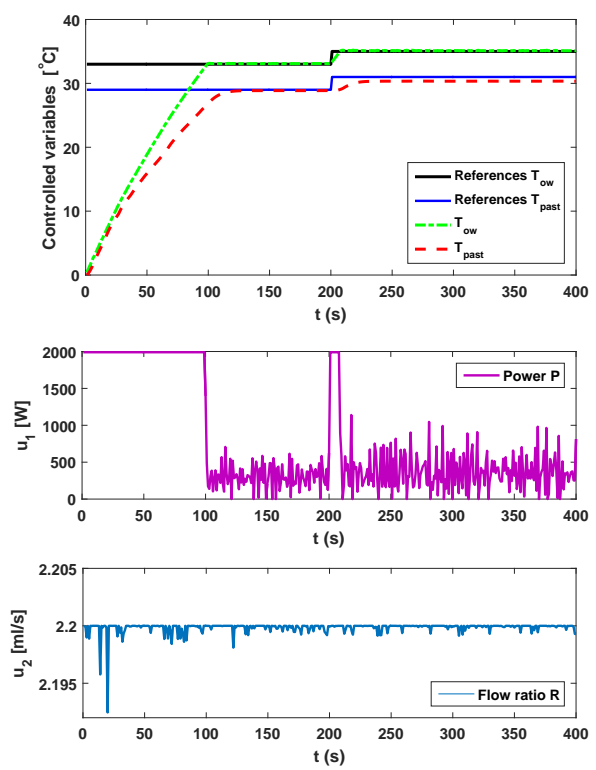

Fig. 4: Control of the output temperatures $\left(T_{\text {ow }}, T_{\text {past }}\right)$ and control inputs, MPC based on quasi-LPV model of the pasteurization plant.

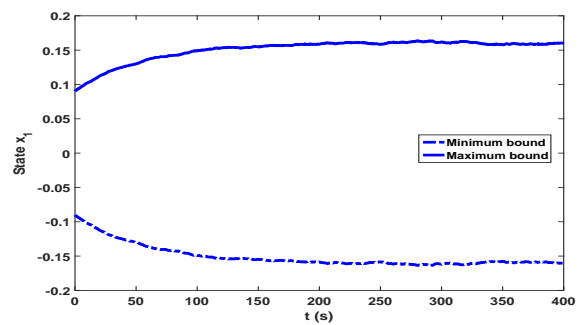

Fig. 5: Uncertainty bounds in the estimated states.

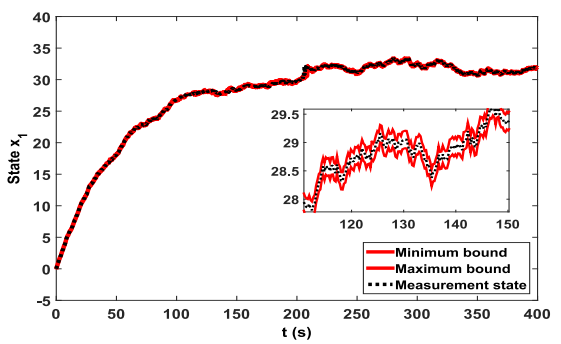

Fig. 6: State under controller.

model, respectively are introduced. The strategy based on the pureLPV model requires that all the varying parameters are scheduled with respect to exogenous variables. In this way, a LTV is obtained and the resulting MPC optimization problem can be solved using efficient quadratic programming solvers. On the other hand, when some of the endogenous variables are used as scheduling variables, the model can be expressed as a quasi-LPV one. The resulting optimization problem becomes non-linear and quite involved to solve. The paper proposes an heuristic strategy based on assuming that the varying parameters do not vary significantly in the prediction horizon. Finally, in the last approach, by bounding the parameter variations with the operating point and considering this variation as 
TABLE I: Comparison of each strategies configuration based on using assumption. $\checkmark$ : assumed, $\times$ : not assumed.

\begin{tabular}{cccc}
\hline Configuration & Assumption 1 & Assumption 2 & Assumption 3 \\
\hline pure-LPV & $\checkmark$ & $\checkmark$ & $\times$ \\
quasi-LPV & $\times$ & $\times$ & $\checkmark$ \\
robust MPC & $\times$ & $\times$ & $\times$ \\
\hline
\end{tabular}

TABLE II: Comparison of some criteria of each strategies configuration. 1: best performance, 3: worst performance.

\begin{tabular}{|c|c|c|c|c|c|c|c|c|c|}
\hline \multirow[t]{2}{*}{ Ranking } & \multicolumn{2}{|c|}{ Faster system response } & Without overshoot & \multicolumn{2}{|c|}{ Less steady-state errors } & \multicolumn{2}{|c|}{ Less conservativeness } & \multicolumn{2}{|c|}{ Non Assumption } \\
\hline & $T_{o w}$ & $T_{\text {past }}$ & $T_{o w} \quad T_{p a s t}$ & $T_{o w}$ & $T_{\text {past }}$ & $T_{o w}$ & $T_{\text {past }}$ & $T_{o w}$ & $T_{\text {past }}$ \\
\hline 1 & pure-LPV & pure-LPV & quasi-LPV & pure-LPV & pure-LPV & pure-LPV & pure-LPV & Robust MPC & Robust MPC \\
\hline 2 & quasi-LPV & Robust MPC & Robust MPC quasi-LPV & quasi-LPV & quasi-LPV & quasi-LPV & quasi-LPV & quasi-LPV & quasi-LPV \\
\hline 3 & Robust MPC & quasi-LPV & pure-LPV Robust MPC & Robust MPC & Robust MPC & Robust MPC & Robust MPC & pure-LPV & pure-LPV \\
\hline
\end{tabular}
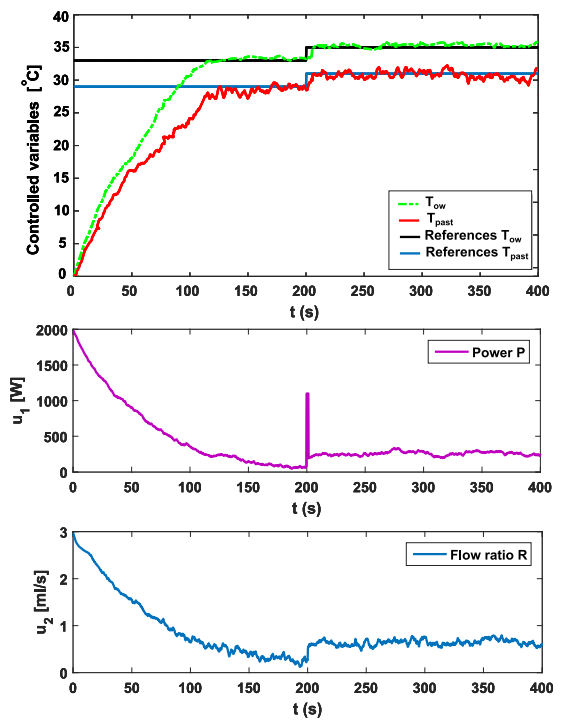

Fig. 7: Control of the output temperatures $\left(T_{o w}, T_{\text {past }}\right)$ and control inputs using robust MPC based on the LPV model of the pasteurization plant.

model uncertainty, a robust MPC controller is designed. The three strategies are implemented and tested in simulation using a highfidelity model of the pasteurization plant. The simulation results are presented showing that the controlled outputs (temperatures) in all the cases achieve the set-points. Finally, the comprehensive comparison between the performance of the different strategies is presented. According to Tables I and II, it can be conclude that more knowledge about the system and sequence of scheduling variable in LPV model achieved better performance in results. As future research, the proposed approaches will be tested in the real pilot pasteurization plant.

\section{ACKNOWLEDGMENT}

This work has been partially funded by the Spanish Government (MINECO) through the projects ECOCIS (ref. DPI2013- 48243-C21-R), and HARCRICS (ref. DPI2014-58104-R FEDER).

\section{REFERENCES}

[1] S. Qin and T. A. Badgwell, "A survey of industrial model predictive control technology," Control Engineering Practice, vol. 11, no. 7, pp. 733-764, 2003.
[2] D. Hrovat, S. Di Cairano, H. E. Tseng, and I. V. Kolmanovsky, "The development of model predictive control in automotive industry: A survey," in IEEE International Conference on Control Applications (CCA). IEEE, 2012, pp. 295-302.

[3] M. Mirzaei, N. K. Poulsen, and H. H. Niemann, "Model predictive control of a nonlinear system with known scheduling variable," in Proceedings of the 17th Nordic Process Control Workshop. Technical University of Denmark, 2012, pp. 163-168.

[4] W. J. Rugh and J. S. Shamma, "Research on gain scheduling," Automatica, vol. 36, no. 10, pp. 1401-1425, 2000.

[5] J. Mohammadpour and C. Scherer, Control of Linear Parameter Varying Systems with Applications. New York: Springer, 2012.

[6] D. Rotondo, F. Nejjari, and V. Puig, "Robust state-feedback control of uncertain LPV systems: An LMI-based approach," Journal of the Franklin Institute, vol. 351, no. 5, pp. 2781-2803, 2014.

[7] Z. He and L. Zhao, "Quadrotor trajectory tracking based on quasilpv system and internal model control," Mathematical Problems in Engineering, vol. 2015, 2015.

[8] M. V. Kothare, V. Balakrishnan, and M. Morari, "Robust constrained model predictive control using linear matrix inequalities," Automatica, vol. 32, no. 10, pp. 1361-1379, 1996.

[9] N. Wada, K. Saito, and M. Saeki, "Model predictive control for linear parameter varying systems using parameter dependent Lyapunov function," in Circuits and Systems, 2004. MWSCAS'04. The 2004 47th Midwest Symposium on, vol. 3. IEEE, 2004, pp. iii-133.

[10] E. Garone and A. Casavola, "Receding horizon control strategies for constrained LPV systems based on a class of nonlinearly parameterized Lyapunov functions," IEEE Transactions on Automatic Control, vol. 57, no. 9, pp. 2354-2360, 2012.

[11] A. Rosich and C. Ocampo-Martinez, "Real-time experimental implementation of predictive control schemes in a small-scale pasteurization plant," in Developments in Model-Based Optimization and Control. Springer, 2015, pp. 255-273.

[12] S. Niamsuwan, P. Kittisupakorn, and I. M. Mujtaba, "Control of milk pasteurization process using model predictive approach," Computers \& Chemical Engineering, vol. 66, pp. 2-11, 2014.

[13] Armfield, Process Plant trainer PTC23-MKII, Instruction Manual, 2015.

[14] J. Ibarrola, J. Guillén, J. Sandoval, and M. García-Sanz, "Modelling of a high temperature short time pasteurization process," Food Control, vol. 9, no. 5, pp. 267-277, 1998.

[15] J. J. Ibarrola, J. M. Sandoval, M. García-Sanz, and M. Pinzolas, "Predictive control of a high temperature-short time pasteurisation process," Control Engineering Practice, vol. 10, no. 7, pp. 713-725, 2002.

[16] T. Alamo, J. M. Bravo, and E. F. Camacho, "Guaranteed state estimation by zonotopes," Automatica, vol. 41, no. 6, pp. 1035-1043, 2005.

[17] W. Kühn, "Rigorously computed orbits of dynamical systems without the wrapping effect," Computing, vol. 61, no. 1, pp. 47-67, 1998. 